\title{
Sex, Age, and Cognitive Correlates of Asymmetries in Thickness of the Cortical Mantle Across the Life Span
}

\author{
Kerstin J. Plessen, ${ }^{1,2,3}$ Kenneth Hugdahl, ${ }^{4,5,6}$ Ravi Bansal, ${ }^{7,8}$ Xuejun Hao, ${ }^{7,8}$ and Bradley S. Peterson ${ }^{7,8}$ \\ ${ }^{1}$ Center for Child and Adolescent Mental Health, Capital Region Psychiatry, Denmark, ${ }^{2}$ Department of Clinical Medicine, Faculty of Health Sciences, \\ University of Copenhagen, Denmark, ${ }^{3}$ Department of Clinical Medicine, Faculty of Medicine and Odontology, University of Bergen, Norway, ${ }^{4}$ Department \\ of Biological and Medical Psychology, University of Bergen, Norway, ${ }^{5}$ Division of Psychiatry and the BMH-Centre, Haukeland University Hospital, Bergen, \\ Norway, ${ }^{6}$ Department of Radiology, Haukeland University Hospital, Bergen, Norway, ${ }^{7}$ Center for Developmental Neuropsychiatry, Department of \\ Psychiatry, Columbia University College of Physicians and Surgeons, New York, New York 10032, and ${ }^{8}$ New York State Psychiatric Institute, New York, New \\ York 10032
}

We assessed the correlations of age, sex, and cognitive performance with measures of asymmetry in cortical thickness on high-resolution MRIs in 215 healthy human children and adults, 7-59 years of age. A left $>$ right asymmetry in thickness of the cortical mantle was present throughout the entire lateral, dorsal, and mesial surfaces of the frontal lobe, extending into primary sensory, superior parietal, and anterior superior temporal cortices. A right $>$ left asymmetry was present in the lateral, mesial, and dorsal surfaces of the posterior temporal, parietal, and occipital cortices, as well as in the entire inferior surface of the brain. An exaggerated left $>$ right asymmetry was detected in females in anterior brain regions, and an exaggerated right $>$ left asymmetry was detected in males in the orbitofrontal, inferior parietal, and inferior occipital cortices. Weaker moderating effects of sex were scattered along the mesial surface of the brain. Age significantly moderated asymmetry measures in the inferior sensorimotor, inferior parietal, posterior temporal, and inferior occipital cortices. The age $\times$ asymmetry interaction derived from a steeper decline in cortical thickness with age in the right hemisphere than in the left on the lateral surface, whereas it derived from a steeper decline with age in the left hemisphere than in the right on the mesial surface. Finally, measures of performance on working memory and vocabulary tasks improved with increasing magnitudes of normal asymmetries in regions thought to support these cognitive capacities.

Key words: asymmetry; cognitive performance; cortical thickness

\section{Introduction}

One of the most prominent anatomical features of the human brain is its division into two distinct hemispheres, yet few studies have assessed morphological asymmetries and their development across the human life span (Hellige, 1990; Hugdahl and Hirnstein, 2013). Important developmental processes are known to modify cortical architecture to thin the cerebral cortex in support of more efficient information processing and greater specialization of cognitive functions (Gogtay et al., 2004; Tau and Peterson, 2010).

Differing imaging methodologies have yielded seemingly conflicting findings in previous studies of anatomical asymmetries in

Received Aug. 28, 2013; revised March 19, 2014; accepted March 25, 2014.

Author contributions: K.J.P., K.H., and B.S.P. designed research; K.J.P., R.B., and B.S.P. performed research; R.B., X.H., and B.S.P. contributed unpublished reagents/analytic tools; K.J.P., R.B., X.H., and B.S.P. analyzed data; K.J.P., K.H., R.B., and B.S.P. wrote the paper.

This work was supported by the Research Council of Norway, the Western Norway Health Authority, and the K.G. Jebsen Foundation grants to K.J.P. and K.H. and National Institute of Mental Health Grants MH36197, K02-74677, MH068318, and MH59139 to B.S.P. We thank Jun Liu, New York State Psychiatric Institute, New York and Morten Aagaard Petersen, Bispebjerg Hospital, Denmark for statistical expertise.

The authors declare no competing financial interests.

Correspondence should be addressed to Dr. Bradley S. Peterson, 1051 Riverside Drive, Unit 74, New York, NY, 10032. E-mail:bp2014@columbia.edu.

DOI:10.1523/JNEUROSCI.3692-13.2014

Copyright $\odot 2014$ the authors $\quad 0270-6474 / 14 / 346294-09 \$ 15.00 / 0$ healthy adults. Studies using voxel-based morphometry have reported a right $>$ left asymmetry for gray matter extending from prefrontal to temporoparietal regions (Watkins et al., 2001) and a left $>$ right asymmetry in posterior brain regions (Good et al., 2001; Watkins et al., 2001; Takao et al., 2011). One MRI study (Lyttelton et al., 2009) reported a leftward asymmetry in surface area for the planum temporale, Heschl's gyrus, and supramarginal gyrus, and a rightward asymmetry in cortical thickness along the mesial wall.

Region of interest studies have reported larger occipital poles in the left and larger frontal poles in the right hemisphere, although the other asymmetries in regional volumes have been less reliable (Raz et al., 1997, 2004).

Deformation-based surface morphometry is another method to map patterns of asymmetries in the brain. One study in young adults has revealed a significant leftward asymmetry in cortical thickness (CT) in precentral, middle frontal, anterior temporal, and superior parietal regions and a rightward asymmetry in posteroinferior temporal and inferior frontal regions (Luders et al., 2006). A second study of CT in a large longitudinal study of children and adolescents (Shaw et al., 2009) reported a prominent interaction of age with asymmetry, in which a left $>$ right asymmetry in CT of the inferior frontal lobe and a right $>$ left asymmetry in thickness of posterior temporal and occipital re- 


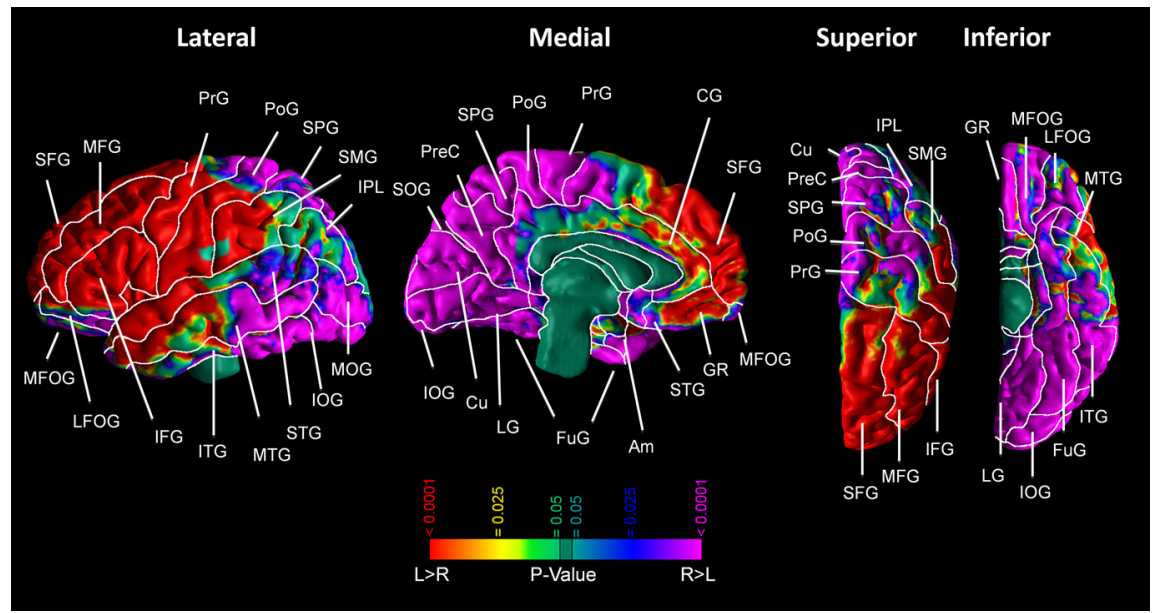

Figure 1. Maps of the main effect of asymmetry $(N=215)$. The color bar indicates the color coding for $p$ values associated with the main effect of asymmetry on CT. Warmer colors (red and yellow) represent positive correlations of cortical thickness with the left hemisphere, indicating regions where the cortex is thicker in the left hemisphere; and cooler colors (blue and purple) represent positive correlations of cortical thickness with the right hemisphere, indicating regions where the cortex is thicker in the right hemisphere. Correlations are visualized on the lateral, medial, superior, and inferior surfaces. These images show voxels that survived correction for multiple comparisons with GRF correction. Cortical regional boundaries indicate the following (Hao et al. 2011): Am, Amygdala; CG, cingulate gyrus; Cu, cuneus; FuG, fusiform gyrus; GR, gyrus rectus; IFG, inferior frontal gyrus; IOG, inferior occipital gyrus; IPL, inferior parietal lobule; ITG, inferior temporal gyrus; LFOG, lateral fronto-orbital gyrus; LG, lingual gyrus; MFG, middle frontal gyrus; MFOG, middle fronto-orbital gyrus; MOG, middle occipital gyrus; MTG, middle temporal gyrus; PreC, precuneus; PoG, postcentral gyrus; PrG, precentral gyrus; SFG, superior frontal gyrus; SMG, supramarginal gyrus; SOG, superior occipital gyrus; SPG, superior parietal gyrus; STG, superior temporal gyrus.

gions reversed following the transition from early childhood to adolescence.

We aimed to resolve some of the discrepant findings from studies of children and adults by assessing the main effects of asymmetry on CT in a single, large sample of healthy children and adult participants. We identified regions of anatomical asymmetry present across participants of all ages as well as interactions of asymmetry with sex and with age. Finally, we intended to explore correlations of asymmetry with two common lateralizing measures of cognitive functioning.

Based on findings from prior studies (Luders et al., 2006; Shaw et al., 2009), we hypothesized significant left $>$ right asymmetries in frontal and anterior temporal cortices and right $>$ left asymmetries in posterior temporal, parietal, and occipital cortices, independent of age of the participant. Moreover, differing findings in youth and adults in those prior studies suggested that we would detect a significant age $X$ asymmetry interaction in inferior frontal and occipital regions. Finally, based on prominent differences across the sexes in language abilities and their purported hemispheric lateralization, we expected that asymmetries would vary with sex. Ours is, to our knowledge, the first report on correlations of CT with age, sex, and asymmetry in a large sample of participants from young childhood to older adulthood.

\section{Materials and Methods}

Participants were recruited randomly from a telemarketing list of nearby households. All participants underwent a standardized diagnostic interview: Schedule for Affective Disorders and Schizophrenia for School-Age Children-Present and Lifetime Version (Kaufman et al., 1997) for children and the Structured Clinical Interview for the DSM-IV Axis I Disorders for adults (Spitzer et al., 1992). Exclusion criteria included a current DSM-IV Axis I disorder or lifetime history of Attention-Deficit/Hyperactivity disorder, tic disorder, Obsessive Compulsive Disorder, substance abuse disorder, psychotic disorder, developmental delay, epilepsy, head trauma with loss of consciousness, or IQ $<80$, as measured by standard tests of intelligence. Written informed consent was obtained from all participants and/or parents, as appropriate for age. We included 215 participants $7-59$ years of age (mean 24.8 years, SD 14.2 years, median 21.7 years), 106 females (mean 25.6 years) and 109 males (mean 24.1 years). Ninety-seven participants were children ( $7-18$ years of age) and 118 were adults (19-59 years of age). Handedness assignment was based on the hand the participants used for writing, which is known to correlate highly with more extensive hand-preference measures (Corey et al., 2001). In addition, we acquired a detailed assessment of handedness in the majority of participants $(N=146)$ using the Edinburgh Handedness Inventory (Oldfield, 1971). A total of 200 participants were primarily right-handed, and 15 primarily left-handed.

Cognitive measures. We explored the correlations of anatomical asymmetry with two cognitive measures in a subgroup of participants to whom the tests were administered: (1) working memory $(N=130)$ was measured with Digit Span (DS; summing raw scores performed forward as an index of auditory attention and backward as an index of verbal working memory capacities and converting to the age-corrected scaled scores) using identical procedures in the WAIS in adults or the "Number" subscale of the Children's Memory Scale in children (both referred to here as the DS; and (2) verbal performance $(N=100)$ was measured with the Vocabulary test in either the WAIS in adults or WISC in children, all within 4 weeks of the MRI scan. We selected these tests for correlation with our asymmetry measures because both DS (Henson et al., 2000) and Vocabulary (Warrington et al., 1986) are considered lateralizing tasks, they have high reliability and age norms that yield comparable scaled scores across age groups (Canivez et al., 2005), and they were acquired using identical procedures across the largest number of participants in our dataset. Unlike prior reports that have explored correlations between measures of IQ and brain structure in each hemisphere separately while taking into account age, sex, and laterality effects (Haier et al., 2004, 2005; Colom et al., 2006; Karama et al., 2009; Schmithorst, 2009; Gläscher et al., 2010; Goh et al., 2011; Margolis et al., 2013; Ziegler et al., 2013), we correlated differences in CT across hemispheres (i.e., asymmetry measures) with performance measures on selected tasks voxelwise across the brain. Male and female participants did not differ in respect to their age-scaled performance on the DS test (females $[N=60]: 10.48, \mathrm{SD}$ 2.42; males $[N=70]: 10.61$, SD 2.39) or Vocabulary test (females $[N=$ 46]: 11.63, SD 3.32; males [ $N=54] 12.43$, SD 3.20). The correlation between the Vocabulary and DS test performances was moderate for both sexes ( $r=0.35$; males $r=0.34$; females $r=0.37$ ), consistent with the loading of both tests on the general intelligence factor (Colom et al., 2006).

MRI scanning. High-resolution MRI images were obtained on a single GE Signa 1.5 Tesla scanner (General Electric), with head positioning standardized using canthomeatal landmarks. T1-weighted anatomical images were acquired using a 3D spoiled gradient echo pulse sequence with repetition time $=24 \mathrm{~ms}$, echo time $=5 \mathrm{~ms}, 45^{\circ}$ flip, frequency encoding superior-to-inferior, no wrap, $256 \times 192$ matrix, field of view $=30 \mathrm{~cm}, 2$ signal averages.

Image processing and statistical analyses. Before processing, images were flipped randomly in the left-right direction to guard against operator bias in definition of each hemisphere. We corrected for large-scale variations in signal intensities across the brain using an automated tool (Sled et al., 1998). The brains of all participants were isolated from nonbrain tissue using an automated tool for brain extraction (Shattuck and Leahy, 2002) combined with manual editing to remove connecting dura on each slice in the sagittal, coronal, and axial views. Cortical gray matter was defined using a combination of thresholding of representative values 
of gray and white matter and subsequent manual editing in all three orthogonal views. Intraclass correlation coefficients for these measures were all $>0.98$.

The previously imposed random flips of the isolated brains were then reversed to provide brains in their native radiological orientation. Each brain was then coregistered to an appropriately selected template brain (see below) using a similarity transformation (Viola and Wells, 1995) (including 3 translation, 3 rotation, and 1 global scaling parameter) to account for variations in head orientation and overall brain volumes. From the coregistered brain of each participant, we subtracted its cortical mantle. We then applied a 3D morphological operator to distance-transform this coregistered brain without the cortex (Rosenfeld and Pfaltz, 1968; Haralick and Shapiro, 1992). This operation calculated cortical thickness as the smallest distance of each point on the pial surface from the outermost surface of the white matter in the coregistered brain. Because cortical thicknesses were measured in template space, their values inherently accounted for generalized scaling effects of the cerebrum, given that the brain and its local features, such as cortical thickness, were already scaled during the similarity transformation to the template brain.

The brains were transected along their interhemispheric fissures using a cubic splines plane fitted to standard midline anatomical landmarks (Press et al., 1992). To construct a measure of asymmetry as a voxelwise subtraction of thickness measures of the right hemisphere from those of the left hemisphere, we first inverted the right hemisphere of each brain along its left-right axis to create a mirror image of itself, so that it now had the general appearance of the left hemisphere of the same brain. We then warped the inverted right hemisphere to the left hemisphere of the same brain using a high-dimensional, nonrigid, fluid-flowbased warping algorithm to produce identical-appearing hemispheres that allowed us to establish a precise labeling of the correspondence of points across their surfaces. Next, the left hemisphere and the identically appearing, inverted right hemisphere of each brain were then rigidly transformed (using three translation and three rotation parameters) to the left hemisphere of the template brain to bring them into close approximation. Both hemispheres were then warped to the exact same size and shape as the left hemisphere of the template brain using a highdimensional, nonrigid, warping algorithm based on the dynamics of fluid flow (Christensen et al., 1994). This high-dimensional warping permitted identification of the points across the surfaces of the left and right hemispheres of each brain that corresponded precisely with points across the surface of the left hemisphere of the template brain and, by extension, with points across the surfaces of both hemispheres for all other brains in the dataset.

Volumes of cortical gray matter. Cortical gray matter regions were defined using the International Consortium for Brain Mapping (ICBM) high-resolution, single-subject template (Mazziotta et al., 2001). This template was generated based on the average of 27 T1-weighted MRI acquisitions in one single subject aligned to the stereotactic space of the ICBM average template (Talairach and Tournoux, 1988). An expert neuroanatomist delineated the cortical gyri manually. This $3 \mathrm{D}$ set of labels was mapped onto the cortical surface to delineate regional boundaries for cortical gray matter (http://www.loni.usc.edu/ICBM/Downloads/ Downloads_ICBMtemplate.shtml).

Statistics. Point correspondences across hemispheres of the same individual and across participants allowed us to use a repeated-measures ANOVA to compare cortical thicknesses voxelwise across hemispheres within and across participants. The repeated measure was CT, and the independent variables were asymmetry (right, left hemisphere), age (in years), and sex (male, female) for every voxel. To accommodate for a curvilinear development of CT with increasing age, we added into the model a quadratic function of age (Shaw et al., 2008), the curvilinear age (the quadratic of age in years ${ }^{2}$ ). All two- and three-way interactions of these variables were considered for inclusion in the model. All models were hierarchically well formulated, with the lower-order terms contributing to significant interactions retained in the model and with nonsignificant interactions and variables eliminated. To depict further the correlation of asymmetry with age, we show regression lines for cortical thickness in voxels from regions where a high degree of asymmetry was identified (dorsolateral prefrontal and occipital cortices) and where prominent asymmetry $\times$ age interactions were detected (cingulate, postcentral, superior temporal, and middle temporal gyri).

We assessed in exploratory analyses the correlation of our measure of asymmetry (left-right CT) with measures of cognitive performance (Vocabulary scores and memory performance) while covarying for age, curvilinear age, and sex. We also assessed these correlations of CT with cognitive performance in the right and left hemisphere, and in males and females separately, while covarying for age, curvilinear age, and sex. We corrected for multiple comparisons using the theory of Gaussian Random Fields (GRFs). The maps display the corrected or the uncorrected $p$ values in the range of 0.0001 to 0.05 (Bansal et al., 2007). Statistically significant results were color-encoded and displayed on the surface of the template of the left hemisphere. For the asymmetry main effect, warm colors (red and yellow) represented left $>$ right thickness and cold colors (blue and purple) represented right $>$ left thickness. For the main effect of sex, warm colors represented CT in females $>$ thickness in males and cold colors indicated CT in males $>$ thickness in females. We used sulcal-based delineations to identify the anatomical locations for our findings on the cortical surface (Hao et al., 2011). 


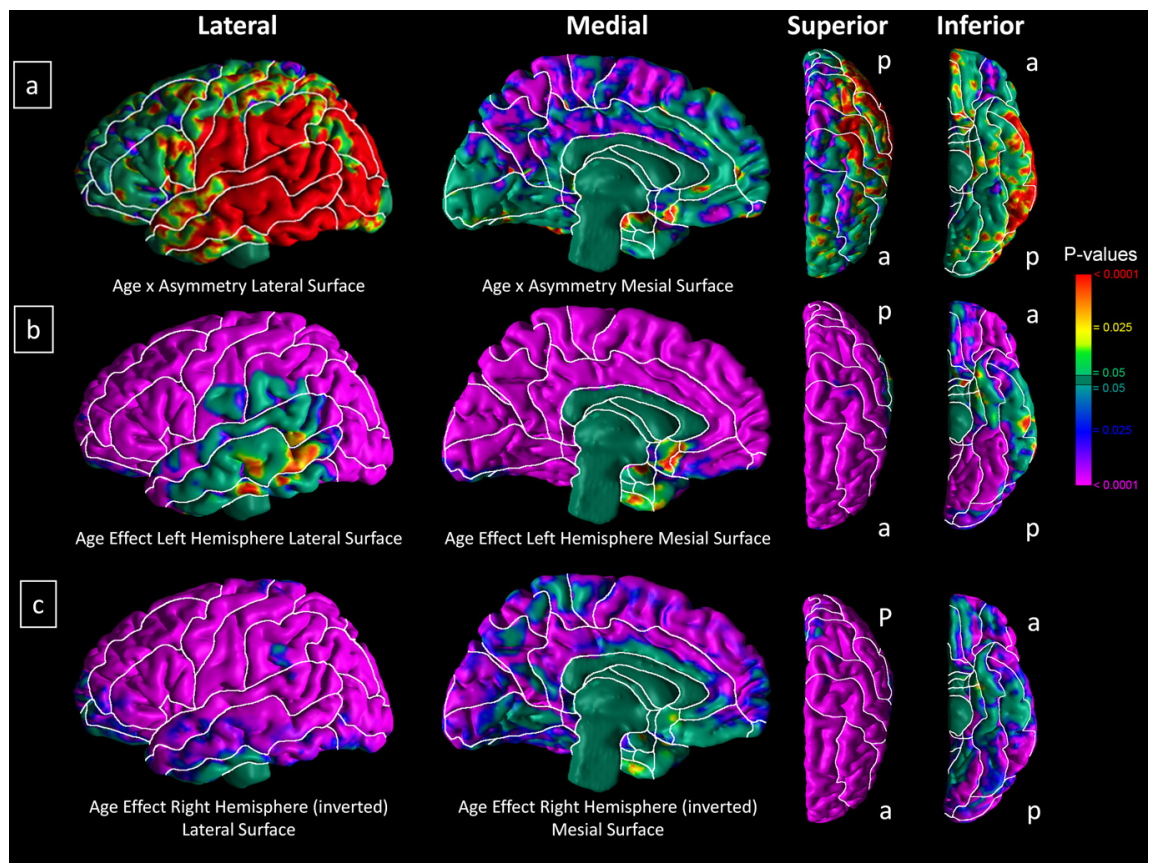

Figure 3. Maps of the age $\times$ asymmetry interaction $(N=215)$. $\boldsymbol{a}$, Interaction effects of age $\times$ asymmetry on cortical thickness, where differing colors (yellow or red vs blue and purple) indicate differing age-dependent effects for the correlations of asymmetry with cortical thickness. $\boldsymbol{b}$, The color bar indicates the color coding of $p$ values associated with the main effect of age for the left hemisphere separately (covaried by sex). $c$, The color bar indicates the color coding of $p$ values associated with the main effect of age for the right hemisphere separately (covaried by sex). The regions of a significant interaction of age $\times$ asymmetry on the lateral surface derive from a decline in cortical thickness with age in the right hemisphere, whereas correlations of cortical thickness with age tend to increase slightly with age in the left hemisphere. The significant age $\times$ asymmetry interactions on the mesial surface derive from a steeper decline in cortical thickness with age in the left hemisphere, thus further accentuating the right $>$ left asymmetry in those cortical regions, including the dorsal and posterior cingulate and the mesial parietal and sensorimotor cortices. a, Anterior; p, posterior.

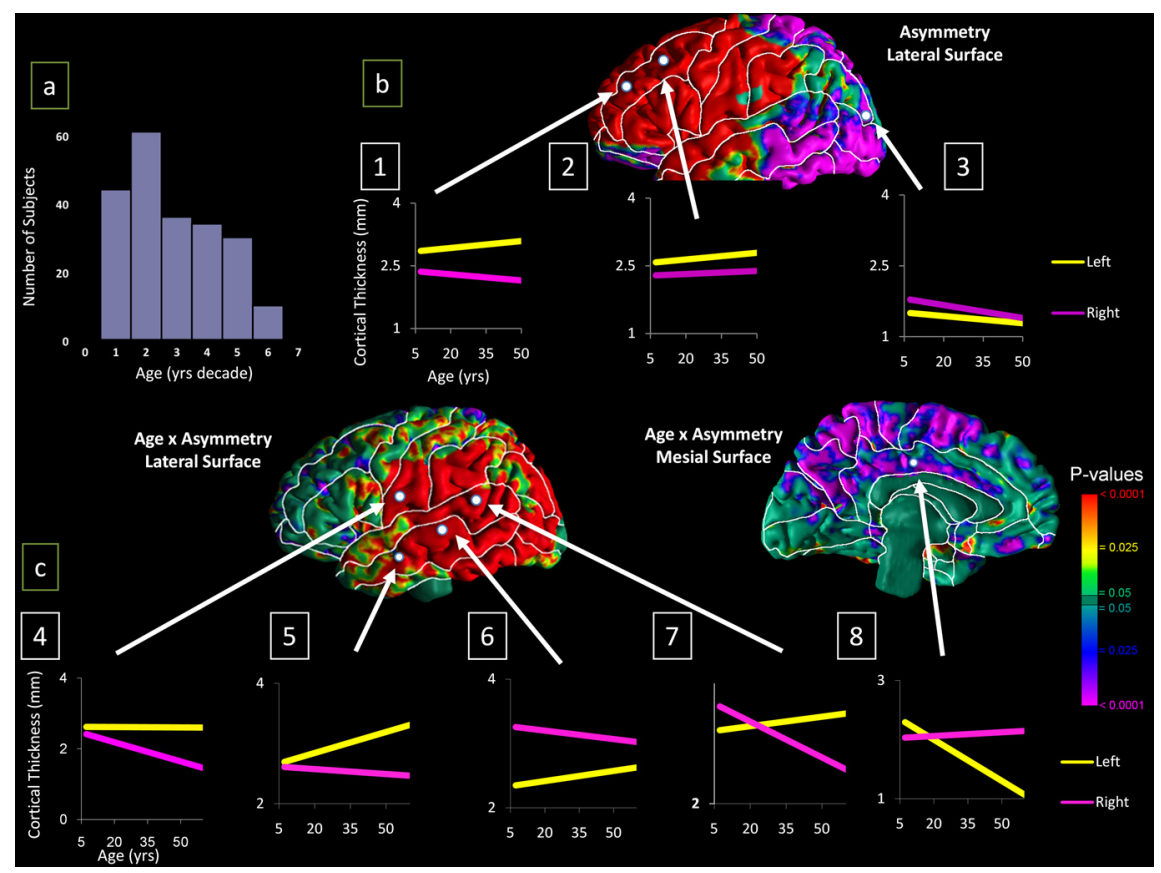

Figure 4. Age distribution and age regressions across life span in selected voxels $(N=215)$. $\boldsymbol{a}$, Distribution of the participants age in years (per decade). $\boldsymbol{b}$, Regression lines indicate correlations of cortical thickness (in millimeters) with age (in years) separately in the left and right hemispheres, both in voxels where we detected a strong asymmetry effect, including dorsolateral prefrontal cortex (panels 1 and 2) and occipital gyrus (panel 3). c, Regression lines indicate the age $\times$ asymmetry interaction for selected voxels (panels 4 -8) on the lateral surface (postcentral, superior temporal, and middle temporal gyri) and on the mesia surface (cingulate gyrus) after controlling for sex.
Finally, cortical gray matter volumes were compared within each gyrus across hemispheres using a repeated-measure analysis (SAS, PROC MIXED), with hemisphere (right and left) and sex (male and female) entered as within-subjects factors while covarying for age. The mean volumes had been adjusted for global scaling effects in the brain of each participant because they were measured in template space, which rescaled each brain to the same volume as the template. We considered all two- and three-way interactions, and statistically nonsignificant terms were eliminated via backward stepwise regression. The model at each step was hierarchically well formulated, so that all component lower-order terms for an interaction were included in the model, regardless of their statistical significance. Moreover, hemisphere, age, and sex were included in the models based on their likely biological relevance.

\section{Results}

The repeated-measures model for CT at each point of the cerebral surface demonstrated significant main effects of asymmetry, sex, age, and age ${ }^{2}$. These effects, however, were moderated significantly by age and sex in a subset of voxels, as indicated by significant two-way asymmetry $\times$ sex and asymmetry $\times$ age interactions. The three-way interaction of age, sex, and asymmetry was not statistically significant anywhere on the cerebral surface.

A map for the main effect of asymmetry (Fig. 1) demonstrated significant left $>$ right CT throughout the entire extent of the lateral, mesial, and dorsal surfaces of the frontal lobe, extending into primary sensory, superior parietal, and anterior superior temporal cortices. We also detected a significant right $>$ left CT asymmetry in the lateral, mesial, and dorsal surfaces of most posterior regions, including posterior temporal, parietal, and occipital cortices, as well as throughout the entire inferior surface of the brain. The exclusion of all left-handed participants did not change these findings (data not shown).

A map for the sex $X$ asymmetry interaction (Fig. 2) showed that sex moderated the CT asymmetry effects in several portions of the brain, including the inferolateral frontal, temporal, and occipital lobes, as well as the entire inferior surface of the brain. Weaker moderating effects of sex were scattered along the mesial surface of the brain. Post hoc analyses (Fig. 2) demonstrated that the sex $X$ asymmetry interaction derived from an exaggerated left $>$ right asymmetry in females in inferior temporal cortices and from an exaggerated 
right $>$ left asymmetry in males in orbitofrontal, inferior parietal, and inferior occipital cortices.

Consistent with a previous report from our laboratory involving a subset of these same participants (Sowell et al., 2003), we detected a prominent inverse correlation of CT with age across the entire dorsal convexity of the brain, including dorsal frontal, parietal, and occipital cortices (Fig. 3). We also detected weak positive curvilinear effects of age in dorsal regions of the brain, which, when combined with a linear decline with age, produced a flattening of the decline in CT in middle and later adulthood. This correlation was in the opposite direction in inferior brain regions, where a weak inverse curvilinear correlation with age combined with a stronger positive linear effect to produce a steep increase and then a flattening correlation of CT with age. These curvilinear effects are consistent with findings previously reported in both cross-sectional and longitudinal samples (Sowell et al., 2003; Shaw et al., 2008). A map of the age $\times$ asymmetry interaction (Fig. 3) demonstrated that age significantly moderated asymmetry effects. In posterior regions, including inferior sensorimotor, inferior parietal, posterior temporal, and inferior occipital cortices, the interaction (depicted in red) derived from cortical thick-

nesses that decreased linearly with age in the right hemisphere but that tended to increase with age in the left hemisphere. These hemisphere-specific age effects produced a left $>$ right asymmetry in older adults (Fig. 3). Along the mesial wall, including the dorsal and posterior cingulate and the mesial parietal and sensorimotor cortices, the interaction (depicted in purple) derived from a more prominent linear decrease in thickness with age in the left hemisphere compared with the right, accentuating in older adults the right $>$ left asymmetry that we detected as a main effect in the entire sample. Correlations of cortical thickness with age differed between voxels where a main effect of asymmetry was located, where regression lines for CT with age were largely parallel across the left and the right hemispheres (Fig. 4, panels 1-3), and voxels where prominent asymmetry $X$ age interaction were located, where the slopes of regression lines for CT with age differed across hemispheres (Fig. 4, panels 4-8).

Exploratory analyses showed that Vocabulary scores correlated inversely with measures of CT asymmetry (left-right thickness) along the lateral surface of the temporal lobe, the posterior portion of the mesial wall, and the entire inferior surface of the brain (Fig. 5, top). Because these were regions where generally a right $>$ left asymmetry was present as a significant main effect (Fig. 1), the inverse correlations indicated that, as this normal right $>$ left asymmetry increased across participants, Vocabulary scores also increased. The significant correlations in these regions derived primarily from inverse correlations of CT with Vocabulary scores in these locations of the left hemisphere (i.e., thinner cortices in the left lateral hemisphere associated with better $\mathrm{Vo}$ cabulary scores) (Fig. 5, bottom). These correlations were significantly stronger in males than in females. In addition, CT correlated positively with Vocabulary scores along the mesial wall of the both hemispheres in both sexes (i.e., thicker cortices associated with better Vocabulary scores), although this correlation was significantly stronger in males along the mesial wall of the right hemisphere (Fig. 5, bottom).

Similar correlations were detected for scores on the DS. Correlations of (left-right) CT asymmetry with DS scores along the posterior mesial surface of the brain differed prominently across males and females, as indicated by an interaction with sex (Fig. 6, top). This interaction derived from stronger positive correlations of CT with DS scores along the posterior mesial surface of the right hemisphere in males than in females (Fig. 6, bottom). This portion of the mesial wall is where normal right $>$ left CT asymmetries were located, and indicates that a more prominent right $>$ left CT in males was associated with better performance on the DS test.

Results of the left-right comparisons using repeatedmeasures analyses for gray matter volumes in cortical ROIs (Table 1) were in general highly consistent with results from deformation-based analyses of CT (Fig. 1), whether asymmetries derived from thicker cortices in the left or right hemisphere (significant left side asymmetries in volume: supramarginal, precentral, middle frontal, inferior frontal, and postcentral gyri; significant right side asymmetries in volume: inferior occipital, precuneus, lingual, and middle occipital gyri). Moreover, the significant asymmetry $\times$ sex interactions (Table 1 ) were similar to those detected using deformation-based analyses (Fig. 1), in that women had larger volumes in gyri where the cortex in deformation-based analyses was thicker on the left (superior and inferior frontal gyri), and men had larger volumes in gyri that 


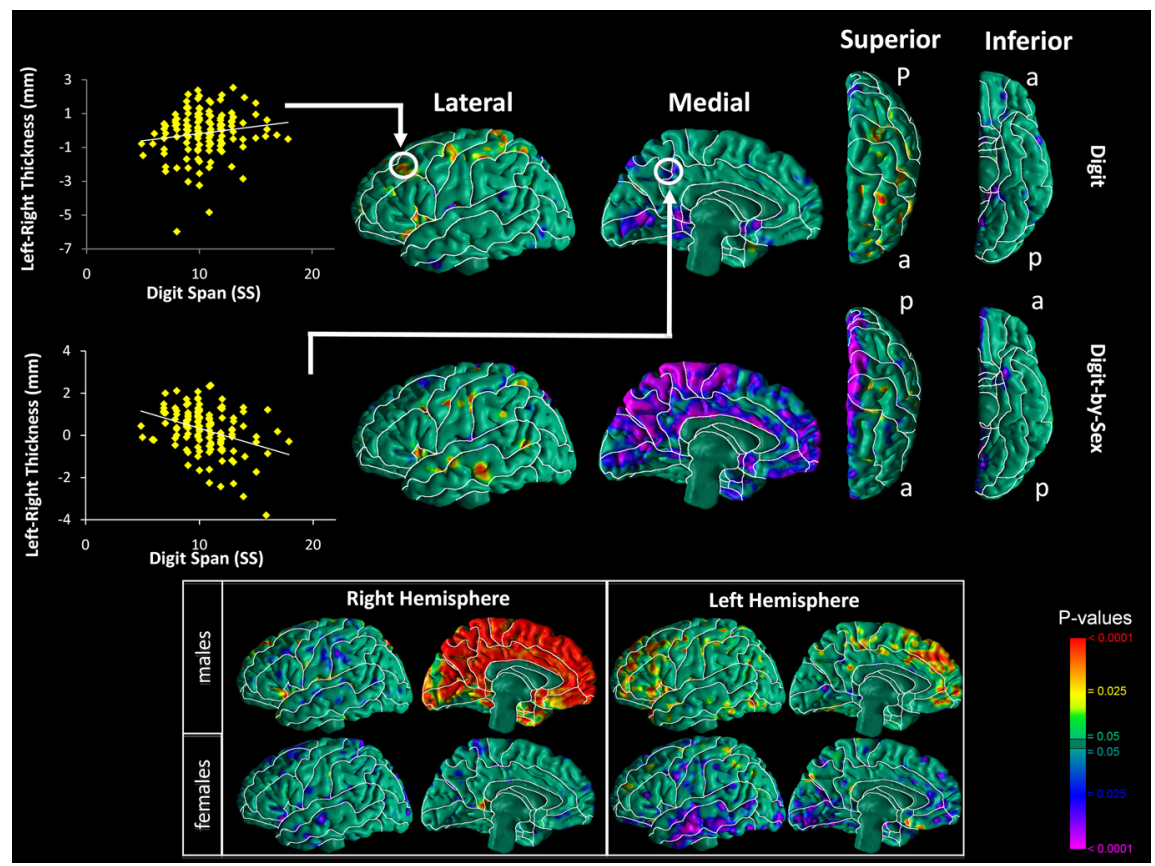

Figure 6. Correlations of $C T$ asymmetry with working memory $(N=130)$. The color bar indicates the color coding for $p$ values associated with the correlations of asymmetry (left hemisphere-right hemisphere cortical thickness) with performance in a memory task (DS scaled score for the DS in adults, "Numbers" subtest of the Children's Memory Scale in children) using the regression equation as follows: Left-right $\mathrm{CT}=\mathrm{Age}+\mathrm{Age}^{2}+\mathrm{Sex}+\mathrm{DS}+\mathrm{DS} \times \mathrm{Sex}$. Warmer colors (yellow and red) represent positive correlations of (T asymmetry with memory performance, and cooler colors (blue and purple) represent inverse correlations of CT asymmetry with performance. The scatterplot, derived from the subtraction of the thickness of left-right CT correlated with the scaled score (SS) of DS (residuals controlled for age and sex), represents the association for every participant. The voxel on the mesial wall is located in the superior parietal cortex, and the voxel on the lateral surface is located in the supramarginal gyrus. Lowest panel, Use of the same model to depict correlations of $\mathrm{CT}$ with memory performance separately for the right and left hemispheres and separately for males $(N=70)$ and females $(N=60)$. a, Anterior; p, posterior.

were thicker on the right (lateral orbitofrontal, fusiform, and inferior temporal gyri).

\section{Discussion}

We have confirmed and substantially extended earlier findings of asymmetry in CT in a large, cross-sectional sample of 215 healthy children and adults. We detected a left $>$ right CT asymmetry across the entire lateral, dorsal, and mesial surfaces of the frontal lobe, extending into primary sensory, superior parietal, and anterior superior temporal cortices. The left $>$ right CT asymmetry in frontal cortices has been reported previously, although in a smaller spatial expanse, in inferior frontal cortices of young children and dorsal frontal cortices of older adolescents (Shaw et al., 2009), and in the middle frontal and precentral gyri of young adults (Luders et al., 2006). A leftward CT asymmetry of the frontal cortex, which includes Broca's region in the inferior frontal gyrus (Broca, 1861; Geschwind and Levitsky, 1968), may correspond anatomically with the functional asymmetry for expressive language in these regions reported in numerous prior human lesion studies and functional MRI and PET studies (Rutten et al., 1999; Horwitz et al., 2003; Hagoort, 2005). Findings of a left $>$ right CT asymmetry in the anterior cingulate, motor, and premotor cortices support findings reported in a prior study using similar methods in young adults (Luders et al., 2006). We also detected a right $>$ left CT asymmetry along the lateral, mesial, and dorsal surfaces of the posterior temporal, parietal, and occipital cortices, and along the entire inferior surface of the brain. Consistent with these findings, thicker cortices in the right hemisphere have been reported previously in young adults in the in- ferior lateral-most portion of the frontal lobe, the posterior inferior temporal cortex, and the posterior mesial wall (Luders et al., 2006). Age significantly moderated CT asymmetry measures in the inferior sensorimotor, inferior parietal, posterior temporal, and inferior occipital cortices. This interaction of age $\times$ asymmetry derived from a steeper decline in CT with age in the right hemisphere than in the left on the lateral surface, whereas on the mesial surface it derived from a steeper decline with age in the left hemisphere than in the right. Age thus accentuated in older adults the normal right $>$ left CT asymmetry detected along the mesial walls of the cerebral hemispheres.

Findings from prior studies that used similar voxelwise analyses of CT across the cerebral surface, when considered at specific ages, are generally consistent with our findings that age significantly moderated CT asymmetry (Luders et al., 2006; Shaw et al., 2009). The age ranges of healthy participants in those two previous studies were restricted either to only children and adolescents (358 participants scanned longitudinally between the ages of 3.2 and 21.8 years) (Shaw et al., 2009) or to only young adults $(N=60$, mean age 25 years) (Luders et al., 2006). Our study thus included the widest age range and assessed age correlations across those ages in a large sample of participants, from young childhood to mature adulthood. The interaction of age with CT asymmetry in posterior brain regions in our study resemble most the findings of relative thickening in left posterior temporal cortices with age reported in the large, longitudinal study of young children through older adolescence (Shaw et al., 2009). The reversal of a right $>$ left asymmetry in posterior temporal and parietal cortices in younger participants to a left $>$ right asymmetry in older participants seems to be a consequence of age-related thinning of the right hemisphere and an agerelated, relative thickening (slowed thinning) of the left hemisphere. The functional relevance of this changing asymmetry with advancing age is unknown (Holland et al., 2007). A right > left asymmetry was noted along the posterior mesial wall in the prior study of young adults (Luders et al., 2006), but unfortunately asymmetries in these mesial regions were not reported in the longitudinal study of children and adolescents (Shaw et al., 2009); therefore, we cannot infer from these prior studies the likely developmental trajectory of the right $>$ left asymmetry in this region. We found in our study, however, that age accentuated in older adults the right $>$ left asymmetry detected as a main effect along the mesial wall in our sample.

Sex of the participants also significantly moderated our findings of CT asymmetries. The left $>$ right CT asymmetry we detected as a main effect in anterior brain regions was significantly more prominent in females, whereas the right $>$ left asymmetry in posterior brain regions was more prominent in males. These sex differences may represent the neuroanatomical correlates of the most robust and best replicated differences in cognitive abilities across sexes, a female advantage on verbal tasks (Kimura and 
Table 1. Volumes of cortical gray matter ${ }^{a}$

\begin{tabular}{|c|c|c|c|c|c|c|c|c|}
\hline \multirow[b]{2}{*}{ Gyri } & \multirow[b]{2}{*}{ Sex } & \multicolumn{2}{|l|}{ Volume } & \multirow[b]{2}{*}{ Asymmetry, $p(F)$} & \multirow[b]{2}{*}{ Sex, $p(F)$} & \multirow[b]{2}{*}{ Age, $p(F)$} & \multirow[b]{2}{*}{ Asymmetry $\times \operatorname{sex}, p(F)$} & \multirow[b]{2}{*}{ Asymmetry $\times$ age, $p(F)$} \\
\hline & & Right, $\mathrm{mm}^{3}$ (SE) & Left, $\mathrm{mm}^{3}$ (SE) & & & & & \\
\hline \multirow[t]{2}{*}{$\mathrm{PH}$} & M & $1803(60.41)$ & $1742(38.84)$ & & & & & \\
\hline & $\mathrm{F}$ & $1755(61.25)$ & 1854 (39.37) & NS & NS & $<0.0001$ (15.39) & $0.0448(4.08)$ & NS \\
\hline \multirow[t]{2}{*}{ SMG } & M & $10,427(276.54)$ & $12,710(212.24)$ & & & & & \\
\hline & $\mathrm{F}$ & $11,217(278.70)$ & $13,499(215.05)$ & $0.0003(13.51)$ & $0.009(6.95)$ & $<0.0001$ (56.34) & NS & $0.045(4.05)$ \\
\hline \multirow[t]{2}{*}{ SFG } & M & $23,595(599.48)$ & $26,610(459.71)$ & & & & & \\
\hline & $\mathrm{F}$ & $24,365(607.72)$ & $29,267(465.92)$ & $<0.0001(101.56)$ & $0.0092(6.91)$ & $<0.0001(101.18)$ & $0.0172(5.77)$ & NS \\
\hline \multirow[t]{2}{*}{ PG } & M & 8903 (192.74) & $10,748(162.44)$ & & & & & \\
\hline & $\mathrm{F}$ & $9827(194.46)$ & $11,672(164.48)$ & $<0.0001(143.52)$ & $<0.0001$ (17.08) & $<0.0001$ (169.42) & NS & NS \\
\hline \multirow[t]{2}{*}{ CG } & M & $15,339(474.99)$ & $15,926(237.95)$ & & & & & \\
\hline & $\mathrm{F}$ & $16,583(476.58)$ & $17,171(241.11)$ & NS & $<0.0001$ (13.65) & $<0.0001(104.99)$ & NS & NS \\
\hline \multirow[t]{2}{*}{ MFG } & M & $11,243(328.12)$ & 14,179 (225.79) & & & & & \\
\hline & $\mathrm{F}$ & $12,346(330.12)$ & $15,281(228.69)$ & $<0.0001(101.13)$ & $0.0005(12.31)$ & $<0.0001(127.85)$ & NS & NS \\
\hline \multirow[t]{2}{*}{ STG } & M & $15,226(302.94)$ & $17,456(271.15)$ & & & & & \\
\hline & $\mathrm{F}$ & $16,087(305.96)$ & $18,316(274.52)$ & $0.0129(6.28)$ & $0.0213(5.38)$ & $<0.0001$ (35.83) & NS & $<0.01(6.81)$ \\
\hline \multirow[t]{2}{*}{ IFG } & M & $13,627(428.80)$ & $15,148(230.36)$ & & & & & \\
\hline & $\mathrm{F}$ & $13,332(434.76)$ & 16,349 (233.47) & $<0.0001(53.87)$ & NS & $<0.0001$ (81.04) & $0.0164(5.85)$ & NS \\
\hline \multirow[t]{2}{*}{ IOG } & M & 2812 (181.62) & 2321 (153.37) & & & & & \\
\hline & $\mathrm{F}$ & 2778 (183.29) & $2286(155.35)$ & $0.0003(13.67)$ & NS & $0.0014(10.41)$ & NS & NS \\
\hline \multirow[t]{2}{*}{$\mathrm{Cu}$} & M & 3290 (168.49) & $3125(147.70)$ & & & & & \\
\hline & $\mathrm{F}$ & 3297 (170.22) & 3132 (149.67) & NS & NS & $<0.0001(27.70)$ & NS & NS \\
\hline \multirow[t]{2}{*}{ LFOG } & M & $4775(214.65)$ & $4322(90.29)$ & & & & & \\
\hline & $\mathrm{F}$ & $4149(217.65)$ & 4514 (91.51) & NS & NS & $<0.0001$ (66.22) & $0.0049(8.9)$ & NS \\
\hline \multirow[t]{2}{*}{ SPG } & M & 7238 (174.12) & 7508 (164.10) & & & & & \\
\hline & $\mathrm{F}$ & 7626 (176.08) & 7896 (166.18) & $0.0155(5.95)$ & NS & $<0.0001(74.16)$ & NS & NS \\
\hline \multirow[t]{2}{*}{ PreC } & M & 6580 (195.72) & $6231(139.76)$ & & & & & \\
\hline & $\mathrm{F}$ & 6903 (197.05) & 6554 (141.62) & $0.0249(5.10)$ & NS & $<0.0001(82.87)$ & NS & NS \\
\hline MTG & M & $14,302(493.13)$ & $14,826(349.99)$ & & & & & \\
\hline & $\mathrm{F}$ & 14,076 (499.76) & 16,121 (354.69) & NS & NS & $0.0002(14.15)$ & $0.0355(4.48)$ & $0.0062(7.65)$ \\
\hline LG & M & 7541 (385.47) & $6361(240.58)$ & & & & & \\
\hline & $\mathrm{F}$ & 7807 (387.50) & 6627 (243.82) & $0.0002(14.90)$ & NS & $<0.0001(22.50)$ & NS & NS \\
\hline PoG & M & $14,380(304.03)$ & $16,534(263.57)$ & & & & & \\
\hline & $\mathrm{F}$ & $15,325(306.95)$ & 17,479 (266.94) & $<0.0001(93.70)$ & $0.011(6.67)$ & $<0.0001(75.04)$ & NS & NS \\
\hline MFOG & M & 5908 (289.48) & 5416 (125.88) & & & & & \\
\hline & $\mathrm{F}$ & 5150 (293.51) & 5759 (127.57) & NS & NS & $<0.0001(28.05)$ & $0.0056(7.84)$ & NS \\
\hline$G R$ & M & $3548(115.46)$ & 3779 (861.42) & & & & & \\
\hline & $\mathrm{F}$ & 3782 (115.88) & 4013 (62.22) & $0.037(4.42)$ & $0.007(7.39)$ & $<0.0001(23.48)$ & NS & NS \\
\hline FuG & $M$ & 7849 (432.72) & $6643(135.33)$ & & & & & \\
\hline & $\mathrm{F}$ & 7137 (438.77) & 7179 (137.15) & $0.0496(3.9)$ & NS & $<0.0001(18.18)$ & $0.0356(4.47)$ & NS \\
\hline EntC & M & $314(14.44)$ & 341 (12.37) & & & & & \\
\hline & $\mathrm{F}$ & 341 (14.58) & $368(12.54)$ & $0.0025(9.37)$ & NS & NS & NS & NS \\
\hline MOC & M & 3371 (197.88) & 3221 (187.11) & & & & & \\
\hline & $\mathrm{F}$ & $3287(200.10)$ & 3138 (189.45) & $0.0171(5.77)$ & NS & $<0.0001(20.97)$ & NS & $0.0377(4.37)$ \\
\hline ITG & M & $10,423(458.82)$ & 9094 (194.30) & & & & & \\
\hline & $\mathrm{F}$ & 9368 (465.22) & 9920 (196.92) & NS & NS & $0.0097(6.81)$ & $0.0066(7.52)$ & NS \\
\hline Subc & $M$ & $1168(46.69)$ & $1068(22.03)$ & & & & & \\
\hline & $\mathrm{F}$ & $1017(47.34)$ & $1081(22.33)$ & NS & NS & NS & $0.0118(6.45)$ & NS \\
\hline
\end{tabular}

${ }^{a}$ Shown are estimates of the mean volumes (in $\mathrm{mm}^{3}$ ) and SE for gray matter in individual gyri of the right and left hemispheres at mean age 24.8 years $(N=215)$. Volumes were compared within each gyrus across hemispheres using a repeated-measures ANOVA (SAS, PROC MIXED) with hemisphere (right, left) and sex (male, female) as within-subjects factors while covarying for age. The results are highly consistent with our deformation-based analyses of cortical thickness. PH, Parahippocampal gyrus; NS, not significant; $S M G$, supramarginal gyrus; $S F G$, superior frontal gyrus; $\mathrm{PG}$, precentral gyrus; $\mathrm{CG}$, cingulate gyrus; $\mathrm{MFG}$, middle frontal gyrus; $\mathrm{STG}$, superior temporal gyrus; IFG, inferior frontal gyrus; IOG, inferior occipital gyrus; Cu, cuneus; LFOG, lateral fronto-orbital gyrus; SPG, superior parietal gyrus; PreC, precuneus; MTG, middle temporal gyrus; LG, lingual gyrus; PoG, postcentral gyrus; MFOG, middle fronto-orbital gyrus; GR, gyrus rectus; FuG, fusiform gyrus; EntC, entorhinal cortex; MOC, middle occipital gyrus; ITG, inferior temporal gyrus; Subc, subcallosal cortex.

Clarke, 2002) and a male advantage on visuospatial tasks (Hines, 2011; Alexander and Wilcox, 2012). Functional imaging studies have shown that language functions involve anterior brain regions most prominently (Binder and Price, 2001) and visuospatial functions involve posterior brain regions most prominently (Hugdahl et al., 2006), consistent with the exaggerated cortical asymmetries we identified in females in anterior brain regions and in males in posterior brain regions. Sexually dimorphic cognitive abilities have been hypothesized to have evolutionary origins, with male-specific demands for hunting early in our species conferring an advantage for those with better visuospatial abilities, and more female-specific demands for socialization and child-rearing conferring an advantage for those with superior language abilities (Hines, 2011; Alexander and Wilcox, 2012).
Sex-specific differences in cortical asymmetries therefore could have a similar origin. Cytoarchitectonic mapping in postmortem brains is required to define the underlying microstructural determinants of the asymmetries in CT that we have identified (Amunts and Zilles, 2001).

The degree of hemispheric asymmetry in our participants correlated significantly with measures of performance on two lateralizing cognitive tasks, Vocabulary (assessing verbal performance) and DS (assessing working memory), independent of age. Inverse correlations of Vocabulary scores with the magnitude of left-right CT asymmetries were located in the inferior and posterior temporal cortices and along the inferior surface of the brain. The direction of correlations suggested that better Vocabulary scores accompanied smaller values of the left-right differ- 
ence measures, or equivalently, greater prominence of the normal right $>$ left asymmetries in these regions; therefore, the more prominent the normal asymmetry in these regions, the better were the Vocabulary scores. This finding was centered on the middle temporal gyrus, which human lesion and stimulation studies have shown supports receptive language functions and the accessing of lexical and semantic information (Dronkers et al., 2004; Hoffman et al., 2012). This region also overlaps with a region in the temporal lobe where earlier studies reported that gray matter volumes correlated with a general factor of intelligence and Full Scale IQ in young adults (Haier et al., 2004; Colom et al., 2006), probably because of the region's role in integrating verbal, visuospatial, working memory, and executive function processes.

The magnitude of asymmetry in our study also correlated inversely along the mesial walls with measures of both forward and backward performance on the DS test, indices of auditory attention and verbal working memory capacities, respectively. The direction of the correlation indicated that exaggeration of the normal right $>$ left asymmetry in these regions was associated with better task performance. The mesial posterior parietal cortex is one of the regions that activate most strongly during working memory tasks (Owen et al., 2005). DS scores also correlated positively with CT asymmetry in scattered regions located along the dorsolateral portions of the frontal and parietal cortices, the locations of left $>$ right asymmetries in our dataset, indicating again that an exaggeration of normal asymmetry of these cortices accompanied better performance. These portions of the parietal cortex, including the supramarginal gyri, support verbal working memory processes, as suggested initially by reports that lesions to this region produce selective impairment in auditory verbal short-term memory (Warrington et al., 1971) and confirmed in numerous functional imaging studies (Cabeza and Nyberg, 2000). Likewise, involvement of the mid-dorsolateral portions of the frontal cortex in verbal working memory processes has been documented in numerous task-based functional imaging studies (Petrides et al., 1993). We detected prominent sex-specific effects in the correlations of CT with both cognitive measures, especially along the right mesial surface of the brain, where these correlations were strong and spatially extensive in males but almost absent in females.

Human beings have long been fascinated by the mystery of the bicameral brain (Dax, 1865). Why do humans and other animals have two cerebral hemispheres? One obvious reason is the specialization of each hemisphere for processing of sensory information from, and controlling motor behavior in, the contralateral body. Another reason likely is to provide an anatomical and functional reserve in the event of damage to one of the hemispheres. Yet another is to increase the degree of parallel and distributed processing in the brain, speeding and dividing tasks across the two hemispheres. The asymmetries in CT suggest that anatomical differences across the two hemispheres also likely have important additional cognitive and behavioral consequences, a suggestion supported by our findings that more prominent asymmetries are associated with better Vocabulary scores and working memory abilities. The moderating effects of age on cortical asymmetries that we detected suggest that the cognitive and behavioral functions that these anatomical asymmetries support likely change through ontogeny, whereas the moderating effects of sex suggest that these asymmetries may have changed through phylogeny, presumably to support functional specialization of sex-typed behaviors.

\section{References}

Alexander GM, Wilcox T (2012) Sex differences in early infancy. Child Dev 6:400-406.

Amunts K, Zilles K (2001) Advances in cytoarchitectonic mapping of the human cerebral cortex. Neuroimaging Clin North Am 11:151-169. Medline

Bansal R, Staib LH, Plessen KJ, Xu D, Royal J, Peterson BS (2007) Voxelwise comparisons of the morphology of diffusion tensors across groups of experimental subjects. Psychiatry Res 156:225-245. CrossRef Medline

Binder J, Price CJ (2001) Functional neuroimaging of language. In: Handbook of functional neuroimaging of cognition (Kingstone A, ed), pp 187250. Cambridge, MA: Massachusetts Institute of Technology.

Broca P (1861) Remarques sur le siège de la faculté du langage articule, suives d'une observation dlaphemié (perte de la parole). Bull Soc Anat 36:330-357.

Cabeza R, Nyberg L (2000) Imaging cognition II: an empirical review of 275 PET and fMRI studies. J Cogn Neurosci 12:1-47. CrossRef Medline

Canivez GL, Neitzel R, Martin BE (2005) Construct validity of the Kaufman Brief Intelligence Test, Wechsler Intelligence Scale for Children-Third Edition, and Adjustment Scales for Children and Adolescents. J Psychoed Assess 23:15-34. CrossRef

Christensen GE, Rabbitt RD, Miller MI (1994) 3D brain mapping using a deformable neuroanatomy. Phys Med Biol 39:609-618. CrossRef Medline

Colom R, Jung R, Haier R (2006) Finding the g-factor in brain structure using the method of correlated vectors. Intelligence 34:561-570. CrossRef

Corey DM, Hurley MM, Foundas AL (2001) Right and left handedness defined: a multivariate approach using hand preference and hand performance measures. Neuropsychiatry Neuropsychol Behav Neurol 14:144152. Medline

Dax M (1865) Lésions de la moitié gauche de l'encéphale coïncidant avec l'oubli des signes de la pensée (lu au Congrés Méridional tenu à Montpellier en 1836). Gazette Hebdomadaire de Médecine et de Chirurgie 2:259-260.

Dronkers NF, Wilkins DP, Van Valin RD Jr, Redfern BB, Jaeger JJ (2004) Lesion analysis of the brain areas involved in language comprehension. Cognition 92:145-177. CrossRef Medline

Geschwind N, Levitsky W (1968) Human brain: left-right asymmetries in temporal speech region. Science 161:186-187. CrossRef Medline

Gläscher J, Rudrauf D, Colom R, Paul LK, Tranel D, Damasio H, Adolphs R (2010) Distributed neural system for general intelligence revealed by lesion mapping. Proc Natl Acad Sci U S A 107:4705-4709. CrossRef Medline

Gogtay N, Giedd JN, Lusk L, Hayashi KM, Greenstein D, Vaituzis AC, Nugent TF 3rd, Herman DH, Clasen LS, Toga AW, Rapoport JL, Thompson PM (2004) Dynamic mapping of human cortical development during childhood through early adulthood. Proc Natl Acad Sci U S A 101:8174-8179. CrossRef Medline

Goh S, Bansal R, Xu D, Hao X, Liu J, Peterson BS (2011) Neuroanatomical correlates of intellectual ability across the life span. Dev Cogn Neurosci 1:305-312. CrossRef Medline

Good CD, Johnsrude I, Ashburner J, Henson RN, Friston KJ, Frackowiak RS (2001) Cerebral asymmetry and the effects of sex and handedness on brain structure: a voxel-based morphometric analysis of 465 normal adult human brains. Neuroimage 14:685-700. CrossRef Medline

Hagoort P (2005) On Broca, brain, and binding: a new framework. Trends Cogn Sci 9:416-423. CrossRef Medline

Haier RJ, Jung RE, Yeo RA, Head K, Alkire MT (2004) Structural brain variation and general intelligence. Neuroimage 23:425-433. CrossRef Medline

Haier RJ, Jung RE, Yeo RA, Head K, Alkire MT (2005) The neuroanatomy of general intelligence: sex matters. Neuroimage 25:320-327. CrossRef Medline

Hao X, Xu D, Bansal R, Liu J, Peterson BS (2011) An improved representation of regional boundaries on parcellated morphological surfaces. Comput Med Imaging Graph 35:206-219. CrossRef Medline

Haralick R, Shapiro L (1992) Computer and robot vision. Reading, MA: Addison-Wesley.

Hellige JB (1990) Hemispheric asymmetry. Annu Rev Psychol 41:55-80. CrossRef Medline

Henson RN, Burgess N, Frith CD (2000) Recoding, storage, rehearsal and 
grouping in verbal short-term memory: an fMRI study. Neuropsychologia 38:426-440. CrossRef Medline

Hines M (2011) Gender development and the human brain. Annu Rev Neurosci 34:69-88. CrossRef Medline

Hoffman P, Pobric G, Drakesmith M, Lambon Ralph MA (2012) Posterior middle temporal gyrus is involved in verbal and non-verbal semantic cognition: evidence from rTMS. Aphasiology 26:1119-1130. CrossRef

Holland SK, Vannest J, Mecoli M, Jacola LM, Tillema JM, Karunanayaka PR, Schmithorst VJ, Yuan W, Plante E, Byars AW (2007) Functional MRI of language lateralization during development in children. Int J Audiol 46: 533-551. CrossRef Medline

Horwitz B, Amunts K, Bhattacharyya R, Patkin D, Jeffries K, Zilles K, Braun AR (2003) Activation of Broca's area during the production of spoken and signed language: a combined cytoarchitectonic mapping and PET analysis. Neuropsychologia 41:1868-1876. CrossRef Medline

Hugdahl K, Hirnstein M (2013) Cerebral hemispheres: behavior and imaging studies. In: Neuropsychology: science and practice, Vol I (Koffler JM, Baron IS, Greiffenstein MF, eds), pp 95-113. New York: Oxford UP.

Hugdahl K, Thomsen T, Ersland L (2006) Sex differences in visuo-spatial processing: an fMRI-study of mental rotation. Neuropsychologia 44: 1575-1583. CrossRef Medline

Karama S, Ad-Dab'bagh Haier R, Deary I, Lyttelton O, Lepage C, Evans A, Group BDC (2009) Positive association between cognitive ability and cortical thickness in a representative US sample of healthy 6 to 18 yearolds. Intelligence 37:145-155. CrossRef Medline

Kaufman J, Birmaher B, Brent D, Rao U, Flynn C, Moreci P, Williamson D, Ryan N (1997) Schedule for Affective Disorders and Schizophrenia for School-Age Children-Present and Lifetime Version (K-SADS-PL): initial reliability and validity data. J Am Acad Child Adolesc Psychiatry 36:980988. CrossRef Medline

Kimura D, Clarke PG (2002) Women's advantage on verbal memory is not restricted to concrete words. Psychol Rep 91:1137-1142. CrossRef Medline

Luders E, Narr KL, Thompson PM, Rex DE, Jancke L, Toga AW (2006) Hemispheric asymmetries in cortical thickness. Cereb Cortex 16:12321238. CrossRef Medline

Lyttelton OC, Karama S, Ad-Dab'bagh Y, Zatorre RJ, Carbonell F, Worsley K, Evans AC (2009) Positional and surface area asymmetry of the human cerebral cortex. Neuroimage 46:895-903. CrossRef Medline

Margolis A, Bansal R, Hao X, Algermissen M, Erickson C, Klahr KW, Naglieri JA, Peterson BS (2013) Using IQ discrepancy scores to examine the neural correlates of specific cognitive abilities. J Neurosci 33:14135-14145. CrossRef Medline

Mazziotta J, Toga A, Evans A, Fox P, Lancaster J, Zilles K, Woods R, Paus T, Simpson G, Pike B, Holmes C, Collins L, Thompson P, MacDonald D, Iacoboni M, Schormann T, Amunts K, Palomero-Gallagher N, Geyer S, Parsons L, et al. (2001) A probabilistic atlas and reference system for the human brain: International Consortium for Brain Mapping (ICBM). Philos Trans R Soc Lond B Biol Sci 356:1293-1322. CrossRef Medline

Oldfield RC (1971) The assessment and analysis of handedness: the Edinburgh Inventory. Neuropsychologia 9:97-113. CrossRef Medline

Owen AM, McMillan KM, Laird AR, Bullmore E (2005) N-back working memory paradigm: a meta-analysis of normative functional neuroimaging studies. Hum Brain Mapp 25:46-59. CrossRef Medline

Petrides M, Alivisatos B, Meyer E, Evans AC (1993) Functional activation of the human frontal cortex during the performance of verbal working memory tasks. Proc Natl Acad Sci U S A 90:878-882. CrossRef Medline

Press WH, Flannery BP, Teukolsky SA, Vetterling WT (1992) Cubic spline interpolation. In: Numerical recipes in FORTRAN. The art of scientific computing, Ed 2, pp 107-110. Cambridge, United Kingdom: Cambridge UP.
Raz N, Gunning FM, Head D, Dupuis JH, McQuain J, Briggs SD, Loken WJ, Thornton AE, Acker JD (1997) Selective aging of the human cerebral cortex observed in vivo: differential vulnerability of the prefrontal gray matter. Cereb Cortex 7:268-282. CrossRef Medline

Raz N, Gunning-Dixon F, Head D, Rodrigue KM, Williamson A, Acker JD (2004) Aging, sexual dimorphism, and hemispheric asymmetry of the cerebral cortex: replicability of regional differences in volume. Neurobiol Aging 25:377-396. CrossRef Medline

Rosenfeld A, Pfaltz J (1968) Distance functions on digital pictures. Pattern Recognition 1:33-61. CrossRef

Rutten GJ, van Rijen PC, van Veelen CW, Ramsey NF (1999) Language area localization with three-dimensional functional magnetic resonance imaging matches intrasulcal electrostimulation in Broca's area. Ann Neurol 46:405-408. CrossRef Medline

Schmithorst VJ (2009) Developmental sex differences in the relation of neuroanatomical connectivity to intelligence. Intelligence 37:164-173. CrossRef Medline

Shattuck DW, Leahy RM (2002) BrainSuite: an automated cortical surface identification tool. Med Image Anal 6:129-142. CrossRef Medline

Shaw P, Kabani NJ, Lerch JP, Eckstrand K, Lenroot R, Gogtay N, Greenstein D, Clasen L, Evans A, Rapoport JL, Giedd JN, Wise SP (2008) Neurodevelopmental trajectories of the human cerebral cortex. J Neurosci 28 : 3586-3594. CrossRef Medline

Shaw P, Lalonde F, Lepage C, Rabin C, Eckstrand K, Sharp W, Greenstein D, Evans A, Giedd JN, Rapoport J (2009) Development of cortical asymmetry in typically developing children and its disruption in attentiondeficit/hyperactivity disorder. Arch Gen Psychiatry 66:888-896. CrossRef Medline

Sled JG, Zijdenbos AP, Evans AC (1998) A nonparametric method for automatic correction of intensity nonuniformity in MRI data. IEEE Trans Med Imaging 17:87-97. CrossRef Medline

Sowell ER, Peterson BS, Thompson PM, Welcome SE, Henkenius AL, Toga AW (2003) Mapping cortical change across the human life span. Nat Neurosci 6:309-315. CrossRef Medline

Spitzer RL, Williams JB, Gibbon M, First MB (1992) The Structured Clinical Interview for DSM-III-R (SCID). I: History, rationale, and description. Arch Gen Psychiatry 49:624-629. CrossRef Medline

Takao H, Abe O, Yamasue H, Aoki S, Sasaki H, Kasai K, Yoshioka N, Ohtomo K (2011) Gray and white matter asymmetries in healthy individuals aged 21-29 years: a voxel-based morphometry and diffusion tensor imaging study. Hum Brain Mapp 32:1762-1773. CrossRef Medline

Talairach J, Tournoux P (1988) Co-planar stereotaxic atlas of the human brain. New York: Thieme Medical.

Tau GZ, Peterson BS (2010) Normal development of brain circuits. Neuropsychopharmacology 35:147-168. CrossRef Medline

Viola PA, Wells WMI (1995) Alignment by maximization of mutual information. In: Fifth International Conference on Comput Vis (IEEE, ed), pp 16-23. Cambridge, MA.

Warrington EK, Logue V, Pratt RT (1971) The anatomical localisation of selective impairment of auditory verbal short-term memory. Neuropsychologia 9:377-387. CrossRef Medline

Warrington EK, James M, Maciejewski C (1986) The WAIS as a lateralizing and localizing diagnostic instrument: a study of 656 patients with unilateral cerebral lesions. Neuropsychologia 24:223-239. CrossRef Medline

Watkins KE, Paus T, Lerch JP, Zijdenbos A, Collins DL, Neelin P, Taylor J, Worsley KJ, Evans AC (2001) Structural asymmetries in the human brain: a voxel-based statistical analysis of 142 MRI scans. Cereb Cortex 11:868-877. CrossRef Medline

Ziegler G, Dahnke R, Winkler AD, Gaser C (2013) Partial least-squares correlation of multivariate cognitive abilities and local brain structure in children and adolescents. Neuroimage 82:284-294. CrossRef Medline 